Gynäkologe 2010 • 43:7-8

DOI 10.1007/s00129-009-2500-1

Online publiziert: 16. Dezember 2009

๑) Springer-Verlag 2009

\author{
L. Beck ${ }^{1} \cdot$ J. Baltzer ${ }^{2}$ \\ ${ }^{1}$ Frauenklinik, Heinrich-Heine-Universität Düsseldorf \\ 2 Krefeld
}

\title{
Verhalten in kritischen Rechtssituationen (Teil 2)
}

Auch die Problematik der Sectio bei bekannter kindlicher Fehlbildung ( $W$. Henrich, C. Bamberg, J.W. Dudenhausen) macht ein Vorgehen notwendig, das sich nach dem Ausmaß der angeborenen Fehlbildungen richtet. Nicht ausgespart bleibt in diesem Beitrag die Sectio auf dringenden Wunsch der Schwangeren trotz infauster fetaler Prognose. bereit gewesen, ein zweites Heft zu „Verhalten in kritischen Rechtssituationen“" vorzubereiten. Die äußerst positive Resonanz auf die Beiträge des ersten Teils haben uns gezeigt, dass der Informationsbedarf der Kolleginnen und Kollegen in Praxis und Klinik groß ist, zumal gerade in letzter Zeit wesentliche gesetzgeberische Änderungen erfolgt sind, die Erläuterungen durch besonders Erfahrene erforderlich machen, sei es aus ärztlicher oder psychosomatischer Sicht, sei es aus der Sicht der Rechtsmediziner, der Medizinrechtler oder des Staatsanwaltes.

Im Beitrag zu den Möglichkeiten, Grenzen und Risiken der Reproduktionsmedizin (D.A. Beyer, F. Amari, K. Diedrich, A. Schroeer) wird ausführlich zu den Methoden der Embryoselektion unter Einschluss der Präimplantationsdiagnostik Stellung genommen. Dabei wird kritisch angemerkt, dass das seit 1991 bestehende Embryonenschutzgesetz nur einen relativ kleinen Rahmen der Methodenwahl zulässt, sodass eine Gesetzesreform als dringend überfällig erscheint.

Ein besonderes Problem ist die Sectio unterhalb der 26. Schwangerschaftswoche, das heißt im unteren Bereich der Lebensfähigkeit (K. Vetter). Im Hinblick auf die unsichere Prognose ist eine umfassende persönliche und interdisziplinäre Beratung der Eltern notwendig. Das operative und neonatologische Vorgehen durch Erfahrene ist unabdingbar.

\section{(> Das Embryonenschutzgesetz lässt nur einen kleinen Rahmen der Methodenwahl zu}

Ein spezielles Problem stellt die Sectio aus kindlicher Indikation ohne Einwilligung der Angehörigen/des Vaters, zum Beispiel bei dringender medizinischer Indikation dar (F. Wolff). Zumeist erfolgt der Eingriff in Übereinstimmung mit der werdenden Mutter und dem anwesenden Partner oder Angehörigen. Je akuter die Notwendigkeit für eine Entscheidung ist, umso geringer ist die Aufklärungspflicht und umso mehr ist der mutmaßliche Wille der Patientin, die unter der Geburt nur bedingt einsichtsfähig ist, vorauszusetzen.

Ein besonderes Problem stellen die möglichen Wiederbelebungsmaßnahmen nach der Geburt eines Kindes mit Fehlbildungen und begrenzter Lebensaussicht dar (T. Höhn). Es wird deutlich, dass das Abschiednehmen nach dem ersten Kennenlernen ihres Kindes für die Eltern einen dynamischen Prozess darstellt, dessen Ausgang offen und häufig mit erheblichen emotionalen Schwankungen verbunden ist.

Die Neuregelung im Schwangerschaftskonfliktgesetz vom 01.01.2010 macht eine aktuelle Erläuterung erforderlich (S. Almer). Dem Arzt werden bei
Feststellung eines pathologischen fetalen Befundes weitgehende Aufklärungs- und Beratungspflichten auferlegt, einerseits um den Lebensschutz des ungeborenen Kindes zu gewährleisten, andererseits um Spätabtreibungen zu vermeiden.

Nicht unberücksichtigt bleiben dürfen die Probleme, die mit dem Schwangerschaftsabbruch im klinischen Alltag verbunden sind (T. Schwenzer). Auch hier wird der Problembereich aus großer klinischer Erfahrung umfassend erläutert. Im Beitrag „Schwangerschaftsabbruch - Macht sich der ausführende Arzt strafbar?" (R. Stahl) wird deutlich, dass die aktuell geltende Fassung der $\$ \$ 218$ ff einen Rechtsrahmen geschaffen hat, der sowohl den durch die Verfassung geforderten Belangen des Lebensschutzes Rechnung trägt als auch die Möglichkeit eröffnet, in einem rechtlich gesicherten Rahmen die medizinische Unterstützung eines Schwangerschaftsabbruches zu gewährleisten.

\section{( In einer medizinisch- kulturellen Konfliktsituation sind Basiskenntnisse über den kulturell-religiösen Hintergrund notwendig}

Im Beitrag „Der Glaube der Zeugen Jehovas und der ärztliche Heilauftrag - Mögliche Konflikte“ (P. Schelling, C. Lippstreu) wird die Thematik außerordentlich hilfreich dargestellt, da die behandelnde Ärztin bzw. der behandelnde Arzt nicht selten vor einem gravierenden Gewissenskonflikt steht. „Religiöser Glaube - Islam - Mögliche Konflikte im klinisch-gynäkologischen Alltag" ist ein Thema, dem immer mehr Bedeutung zukommt (M. David, I. Llkilic). Es wird deutlich, dass zur 
Lösung medizinisch-kultureller Konflikte Basiskenntnisse über den kulturell-religiösen Hintergrund der Patientin notwendig sind.

Häusliche Gewalt stellt nicht selten ein unausgesprochenes Vergehen dar. Im Beitrag „Schwangere in Not - häusliche Gewalt - Vorgehen bei Verdacht auf Misshandlung bzw. häusliche Gewalt" $(M$. Neises) werden die unterschiedlichen Aspekte körperlichen, sexuellen oder emotionalen Missbrauchs unter Einschluss eines aufmerksamen Untersuchungsbefundes und klaren Regeln für das weitere Vorgehen, zum Beispiel auch im Rahmen der Gesprächsführung dargelegt.

Bei Verdacht auf sexuellen Missbrauch von Kindern (A. Stahl) ist ein angemessener Umgang mit medizinischen Befunden, die als Hinweis auf einen sexuellen Kindsmissbrauch interpretiert werden können, nicht selten eine schwierige Herausforderung. Das weitere Vorgehen im Einzelfall macht eine Abwägung zwischen einem vertrauensvollen Arzt-PatientenVerhältnis einerseits und dem Wohl des möglicherweise von einem Missbrauch betroffenen Kindes erforderlich, auch im Hinblick auf eine Verweigerungshaltung der Eltern. Im Beitrag „Verdacht auf sexuellen Missbrauch - Diagnostik und Befundinterpretation aus rechtsmedizinischer Sicht" (E. Mützel, W. Eisenmen- ger) werden konkrete Hinweise auf notwendige diagnostische Maßnahmen und Aspekte der Befundinterpretation unter Berücksichtigung arztrechtlicher Aspekte erläutert. Gleiches gilt für die Darstellung "Vorgehen bei Verdacht auf Kindstötung“ (E. Mützel, W. Eisenmenger). Dabei sollte nach regelhafter Untersuchung durch den Frauenarzt die abschließende Bewertung von Befunden in enger Zusammenarbeit mit der Rechtsmedizin erfolgen, bedenkt man, dass Kindstötung als minder schwerer Fall des Totschlags gemäß $₫ 213$ StGB gewertet wird.

Der abschließende Beitrag „Zwischen Budget, Haftung und Wohl der Patientin - Ein schwieriger Balanceakt" (D. Berg, K. Ulsenheimer) geht auf die zunehmenden Schwierigkeiten auf diesem Sektor ein, zum Beispiel die Problematik einer Reduktion von Standards, die dadurch bedingt sein kann, dass Kosten eingespart werden müssen. In den letzten Jahren ist zunehmend deutlich geworden, dass eine Ökonomisierung der Medizin als Zeichen der Mittelverknappung anzusehen ist, die zum Ursprung, Mittelpunkt und Maßstab aller Reformbewegungen wird, woraus sich unter dem Etikett der Wirtschaftlichkeit für den Mediziner die im Beitrag genannten gefährlichen Folgen ergeben.
Den Autoren danken wir herzlich, dass sie trotz ihrer Zeitknappheit und beruflichen Belastung bereit waren, auch an diesem Themenheft von „Der Gynäkologe“ mitzuarbeiten. Unser Dank gilt ebenfalls Frau Ursula Hilpert und Frau Dr. Jutta Schneider vom Springer-Verlag, die auch dieses Heft mit großer Umsicht betreut haben. Wir wünschen uns, dass auch dieses Themenheft zum zweiten Teil der Thematik aktuelle Entscheidungshilfe in Praxis und Klinik vermitteln kann.

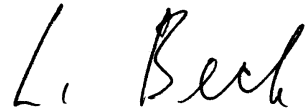

Prof. Dr. L. Beck

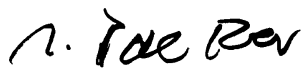

Prof. Dr. J. Baltzer

\section{Korrespondenzadressen}

Prof. Dr. L. Beck

Frauenklinik, Heinrich-Heine-Universität Moorenstraße 5

40227 Düsseldorf

Prof. Dr. J. Baltzer

Jentgesallee 72

47799 Krefeld

prof.baltzer@t-online.de

\section{Exklusiv für Abonnenten :}

\section{Nutzen Sie das Online-Archiv von Der Gynäkologe}

Ihre Vorteile:

- Komfortable und schnelle Recherche nach Themen, Autoren, Suchbegriffen

- Ob unterwegs oder am eigenen PC: Zugriff überall und jederzeit

- Online First: Lesen Sie die aktuellsten Beiträge schon vor Erscheinen des gedruckten Heftes online

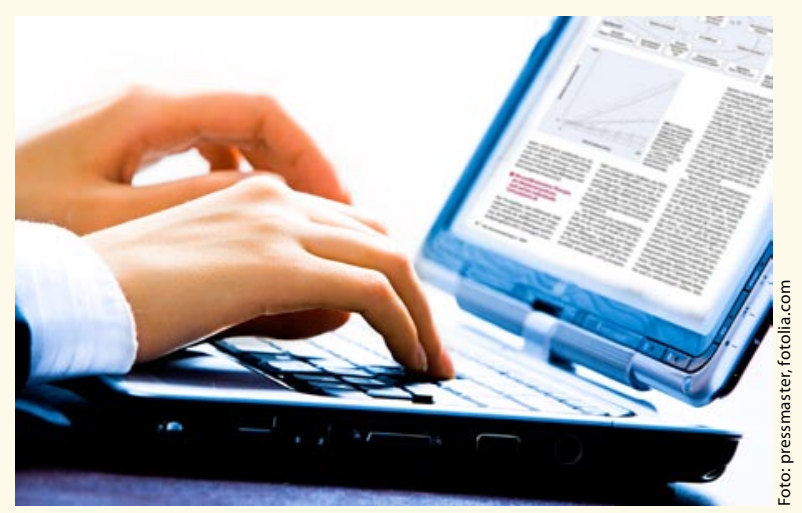

